\title{
Intra-articular injections of platelet-rich plasma releasate reduce pain and synovial inflammation in a mouse model of osteoarthritis
}

\begin{abstract}
Background

Osteoarthritis (OA) is a degenerative joint disease leading to pain and disability for which no curative treatment currently exists. A promising biological treatment for OA is intra-articular administration of platelet rich plasma (PRP). PRP injections in OA joints can relieve pain, although the exact working mechanism is unclear.
\end{abstract}

\section{Purpose}

To examine the effects of PRP on pain, cartilage damage and synovial inflammation in a mouse OA model.

\section{Study design}

Controlled laboratory study.

\section{Methods}

OA was induced unilaterally in the knees of male mice $(n=36)$ by two intra-articular injections of

collagenase at days -7 and -5 . At day 0 , pain was measured by registering weight distribution on the hind limbs; after which mice were randomly divided in two groups. Mice received three intraarticular injections of PRP-releasate (PRPr) or saline in the affected knee. Seven mice per group were euthanized at day 5 to assess early synovial inflammation and cartilage damage. Pain in the remaining mice was registered for a total of three weeks. These mice were euthanized at day 21 to assess cartilage damage and synovial inflammation on histology. Antibodies against iNOS, CD163 and CD206 were used to identify different subtypes of macrophages in the synovial membrane.

\section{Results}

Mice in the PRPr group increased the distribution of weight on the affected joint in two consecutive weeks after start of the treatment $(\mathrm{P}<0.05)$, whereas mice in the saline group did not. 
At day 21, PRPr injected knees had a thinner synovial membrane $(\mathrm{P}<0.05)$, and a trend towards less cartilage damage in the lateral joint compartment $(\mathrm{P}=0.053)$ than saline injected knees. OA knees treated with saline showed less anti-inflammatory (CD206+ and CD163+) cells at day 5 than healthy knees, an observation which was not made in the PRPr-treated group. A higher level of pain at day 7 was associated with a thicker synovial membrane at day 21 . The presence of CD206+ cells was negatively associated with synovial membrane thickness

\section{Conclusions}

In a murine OA model, multiple PRPr injections reduced pain and synovial thickness, possibly through modulation of macrophage subtypes.

\section{Clinical relevance}

PRPr injections shortly after joint trauma can reduce pain and, synovial inflammation and may inhibit OA development in patients. 


\section{What is known about the subject?}

Platelet rich plasma (PRP) is a biological treatment used in human clinical trials for various musculoskeletal diseases, including osteoarthritis (OA). Multiple PRP injections were shown to have beneficial effects on pain and functional impairment, but no effect was found on the severity of radiographic OA. Anti-inflammatory effects of PRP have been demonstrated in vitro on osteoarthritic cartilage and synovium

\section{What this study adds to existing knowledge.}

Here we demonstrate that multiple PRPr injections in a OA model reduce pain and synovial membrane thickness. We provide indications for an effect of PRPr on modulation of synovial macrophage phenotype. This indicates an anti-inflammatory working mechanism of PRPr in vivo. 


\section{INTRODUCTION}

Osteoarthritis (OA) is a degenerative joint disease, characterized by loss of cartilage integrity, changes in subchondral bone, formation of osteophytes and inflammation of the synovial membrane. This process results in pain and disability. Current treatments focus on pain reduction, exercise therapy and - in end-stage OA - joint replacement. To this day no curative treatment exists for OA. Since joint arthroplasties have a limited life-span, the need for diseasemodifying drugs or therapies is high. Ideally such a therapy would inhibit or repair damage to the joint tissues and reduce pain and disability. A biological therapy for tissue injury that has emerged in recent years is treatment with platelet-rich plasma (PRP). PRP is a plasma product extracted from whole blood that contains at least $1.0 \times 10^{6}$ platelets per microliter. ${ }^{29}$ The platelets undergo degranulation after which they release growth factors and cytokines such as transforming growth factor beta (TGF $\beta$ ) and platelet-derived growth factor (PDGF), ${ }^{24,}{ }^{29,}{ }^{37}$ two important factors in tissue healing. PRP can be activated prior to use resulting in PRP-releasate (PRPr), a product containing low amounts of leucocytes and high concentrations of growth factors. ${ }^{9}$ Our group and others have used PRPr previously and have shown that it has antiinflammatory and positive effects on matrix synthesis and accumulation. Furthermore it can induce the chondrogenic properties of chondrocytes. ${ }^{20,47,52} \mathrm{PRPr}$ has been used in a rabbit model and clinically to successfully induce the reparative effect of degenerated intervertebral disc degeneration. ${ }^{1,38}$

Several clinical trials in OA have concluded that multiple PRP injections are safe and have a beneficial effect on OA symptoms such as pain, for up to 12 months. ${ }^{6,17,26,33,34,45}$ Evidence is accumulating from both in vitro and in vivo studies for PRP's potential in the treatment of OA. From preclinical research we know that PRP promotes the proliferation of cells derived from human synovium and cartilage, ${ }^{39,42}$ and that PRP-treated chondrocytes repair cartilage better than non-treated chondrocytes. ${ }^{58}$ These cells in turn produce more superficial zone protein (SZP), which functions as a boundary lubricant that helps to reduce friction and wear. ${ }^{42,43,48}$ PRP itself was also shown to reduce friction in bovine articular cartilage explants. ${ }^{42}$ The anti-inflammatory effects of PRP have been demonstrated both in a co-culture system of osteoarthritic cartilage and synovium $^{42}$ and in human osteoarthritic chondrocytes, where it reduced multiple proinflammatory effects induced by IL- $1 \beta .^{52}$ Furthermore, in a canine OA model, multiple PRP 
injections were shown to have beneficial effects on pain and functional impairment, but no effect on the severity of radiographic OA. ${ }^{11}$

However, while the use of PRP products seems promising for the treatment of OA, the wide variability in outcome parameters evaluated, in models used and in PRP and PRPr production protocols makes interpretation of results between studies difficult. ${ }^{13,}{ }^{22,25}$ This difficultly in comparing the results of PRP research may be one of the reasons why the exact working mechanisms of intra-articular injected PRP products are not fully understood. Unravelling this mechanism could provide an opportunity to further improve therapeutic PRP efficacy.

In this study we assessed the effect of human PRPr compared to saline in a murine model of collagenase-induced OA (CIOA). We studied the effects of PRPr on several OA related processes in the joint: pain, cartilage damage and synovial inflammation and evaluated correlations between these parameters. We payed particular attention to effects on different macrophage phenotypes in the synovium. Our hypothesis is that multiple intra-articular injections of PRPr will reduce pain, have a protective effect on cartilage and inhibit synovial inflammation. 


\section{Materials Methods}

\section{Platelet rich plasma releasate preparation}

Human PRP was acquired from the national blood bank (Sanquin, Amsterdam, The Netherlands) with a platelet count of $8.5 \times 10^{8} / \mathrm{ml}$. This PRP product is produced by pooling buffy coats of 5 different donors in plasma. After centrifugation, the platelets are filtered out to produce the PRP. The blood samples had identical ABO and Rh(D) compatible blood type, and were pathogen free. PRP was activated by adding $10 \% \mathrm{v} / \mathrm{v} 228 \mathrm{mM} \mathrm{CaCl}_{2}$ and incubated on a rotating device at $37^{\circ} \mathrm{C} .^{52}$ After one hour of incubation a clot was formed and the supernatant was harvested. This supernatant contains the released factors of the activated platelets and hence is called the PRP releasate (PRPr). Whereas erythrocytes could be detected in the PRP, they were no longer detected after the activation of the PRP by a clinical cytometer (Model xn1000, Sysmex Netherlands BV, Etten-Leur, Netherlands). Leukocytes were not detectable in the PRP or the PRPr. After harvesting the PRPr the samples were stored at $-80^{\circ} \mathrm{C}$ and used within a week for in vivo experiments. The concentration of PDGF-BB in the cryopreserved samples was $1.2 \times 10^{4} \mathrm{pg} /$ $\mathrm{ml}$, measured by ELISA, confirming that released products were present in the supernatant.

\section{Animal model of injury and treatment}

All animal experiments were performed on 36 male C57/Bl6 mice age 12 weeks (blinded), with approval of the animal ethical committee (blinded 116-14-03). The mice were housed in groups of three or four mice per cage, under 12 hours light-dark cycle at a temperature of $24^{\circ} \mathrm{C}$ degrees Celsius, and had access to water and food ad libitum at the animal testing facilities. Before starting the experiments, mice were allowed to acclimatize for a week. In all mice, OA was induced unilaterally by two intra-articular injections of $6 \mu$ l of 3 units collagenase type VII (Sigma-Aldrich) at days -7 and -5 . Collagenase induced OA is an established model for joint instability in mice. ${ }^{27,44,49,54,55}$ All intra-articular injections were applied under $2.5 \%$ isoflurane anesthesia, with a $50 \mu \mathrm{L}$ glass syringe (Hamilton Company, Ghiroda, Romania) using a 30G needle (Becton, Dickinson and Company, New Jersey, USA). The contralateral control knees were kept naïve and were not injected with any substance. Mice were randomly assigned to either the treatment group with $6 \mu \mathrm{l} \operatorname{PRPr}(\mathrm{n}=11$ mice) or the control group ( $\mathrm{n}=11$ mice) with $6 \mu \mathrm{l}$ saline. Both groups received three consecutive PRPr or saline injections; the first injection was 
given 7 days after the first collagenase injection (referred to as day 0) and repeated with consecutive injections at day 2 and 4 (Figure 1). Weight distribution over the left and right hind limbs was evaluated as an indicator of pain at day 0 and hereafter once weekly for 3 weeks. During the whole experiment the animals were capable to move around freely and reach the food pellets and drink nozzle on the top of their cage. Animals were euthanized at day 21 and knee joints were harvested for histological analysis.

To assess the early effects of PRPr on synovial inflammation, an additional group of animals was used containing 7 mice in each group. These animals underwent identical OA induction and treatment protocols. They were euthanized at day 5 after the start of PRPr treatment and knee joints were prepared for histological evaluation.

\section{Measurement of hind limb weight distribution}

Hind limb weight distribution was registered with an incapacitance tester ${ }^{53}$ (Linton Instrumentation, Norfolk, UK) as an indicator of pain. It is a static method to measure pain, which is validated in mouse, rats and other animal models. ${ }^{3,11,14,15,19,53}$ Mice were positioned on the incapacitance meter with each hind limb resting on a separate force plate. Measurements were performed at day 0, just before therapy administration and hereafter at day 7, 14 and 21.The observer was blinded to the pressures measured during the test. Therefore afterwards, measurements with a registration of below 3 grams ( $<10 \%$ of total bodyweight) per hind limb or less than 10 grams (<30\% of total bodyweight) in total over both hind limbs were excluded. On average 15 measurements per time point per animal were available. For each time point per mouse, the average of these measurements was used to calculate the percentage of weight on the affected limb as an indication of pain in the affected limb. A single value per measurement time point was used for statistical analyses.

\section{Histological analyses}

Knees were fixed in formalin 4\% (v/v) for one week, decalcified in 10\% EDTA for 2 weeks and embedded in paraffin. Coronal sections of $6 \mu \mathrm{m}$ were cut for analysis of synovial inflammation and cartilage damage.

Structural integrity

Cartilage damage was evaluated on thionin-stained sections by two observers blinded to the 
treatment groups with a scoring system described by Glasson et al. ${ }^{16}$ Briefly this score ranges from 0 for normal cartilage, to 6 for cartilage with clefts and erosion to the calcified cartilage in $>75 \%$ of articular surface. For each knee the cartilage quality in the lateral and medial compartment of the knee was scored on 3 sections with $180 \mu \mathrm{m}$ interval between the sections. The maximum and sum score of the 3 sections, for each compartment was taken for analyses. ${ }^{16}$ Data from the lateral compartment is shown, since we used a mild OA model and this was the area where most damage was observed. Thionin stain was used instead of Safranin O because of previously described limitations of the latter, especially regarding sensitivity of GAG depletion., ${ }^{7}$ As an internal check, all knees of the animals terminated at the early time point were stained and scored using both Thionin and Safranin O stain, which showed a very high accordance between both methods (data not shown).

Synovial inflammation

For synovial inflammation assessment, sections were stained with hematoxylin eosin. Images were acquired using the NanoZoomer Digital Pathology program (Hamamatsu Photonics, Ammersee, Germany). Synovial thickness was measured from the capsule to the superficial layer of the synovial membrane in the parapatellar recesses at the medial and the lateral side at three positions per section, based on a previously described method. ${ }^{49}$ These measurements were done on three sections per knee, with $180 \mu \mathrm{m}$ between the sections. In total we obtained 18 synovium thickness measurements per knee, which were averaged to obtain a single value per knee joint.

\section{Macrophage staining}

To evaluate the macrophage subtypes in the synovial membrane, inducible nitric oxide (iNOS) was used as a marker for pro-inflammatory macrophages, cluster of differentiation 163 (CD163) as a marker for anti-inflammatory macrophages, and CD206 as a marker for tissue repair macrophages. For this purpose, sections were deparaffinized, washed and heat-mediated antigen retrieval was performed for $\mathrm{CD} 163$ and $\mathrm{CD} 206$, by placing the slides in $95^{\circ} \mathrm{C}$ citrate buffer $(\mathrm{pH}$ 6) for 20 minutes. Antigen retrieval for iNOS was performed in by placing the slides in $95^{\circ} \mathrm{C}$ Tris-EDTA buffer (pH 9) for 20 minutes. Blocking of aspecific binding was done with 10\% goat serum (Southern Biotech, Birmingham, USA) for 30 minutes. Hereafter, sections were incubated with the primary antibodies iNOS (2.0 $\mu \mathrm{g} / \mathrm{ml} \# 15323$, Abcam, Cambridge, UK), CD163 (0.34 $\mu \mathrm{g} / \mathrm{ml}$, \#182422, Abcam, Cambridge, UK), and CD206 (2.5 g/ml,\#64693, Abcam, Cambridge, UK) for 1 hour, followed by 30 minutes incubation with a biotinylated anti-rabbit Ig link 
(Biogenex, HK-326-UR, Fremont, USA), diluted 1:50 in PBS/1\%BSA. Thereafter, sections were incubated with an alkaline phosphatase-conjugated streptavidin (Biogenex, HK-321-UK) label diluted 1:50 in PBS/1\%BSA. To reduce background, endogenous alkine phosphatase activity was inhibited with levamisole (Sigma-Aldrich Chemie N.V. Zwijndrecht, The Netherlands). Neu Fuchsin (Fisher Scientific, Vienna, Austria) and Napthol AS-MX phosphate (Sigma-Aldrich Chemie N.V. Zwijndrecht, The Netherlands) substrate was used for color development and counterstained with hematoxylin. As a negative control Rabbit IgG antibody (Dako Cytomation, Glostrup, Denmark) was used. The sections were ranked from weakest to strongest staining for either iNOS, CD163, or CD206, by two observers blinded for the treatment group. The maximum rank was based on the total number of joints scored for the individual staining. When multiple sections had similar strength of staining, the mean of the rank numbers was given to each section. Per section the mean rank of both observers was used for analyses.

\section{Statistical Analysis}

For the late time point and pain reduction we consider a decrease in pain of $20 \%$ in the therapy groups to be relevant in our study. According to a power calculation (using a standard deviation of $15 \%$ ), with a statistical power level (1- $\beta$ ) of 0.8 and significance level $(\alpha)$ of 0.05 , our sample size per group for a two tailed hypothesis test was 9 mice. For the short term we considered a $25 \%$ decrease of synovial thickness as relevant for our study. According to a power calculation of the sample size (using a standard deviation of $30 \%$ ), with a statistical power level (1- $\beta$ ) of 0.8 and significance level $(\alpha)$ of 0.05 , our sample size per group for a two tailed hypothesis test was 5 mice. Two additional mice per group gave us $n=11$ for late time point and $n=7$ mice for the early time point. This lead to a total of $n=18$ mice per treatment group and 36 mice in total.

Data was analyzed with IBM SPSS statistics 21 (SPSS, Chicago, IL). For the effect on weight distribution, normality was confirmed with a Shapiro Wilk test. Two-tailed paired t-tests were conducted to evaluate differences between time points within each group and two tailed unpaired t-tests were conducted to evaluate differences between treatment groups at each time point. Statistic testing on synovial thickness measurements where conducted using a Welch t-test, after normality was confirmed with a Shapiro-Wilk test for knees at both day 5 and day 21. To compare the maximum OA scores between saline and PRPr treated groups, a Mann-Whitney U test was performed. A Kruskal-Wallis test was performed for the ranked macrophage data 
between the healthy control, saline and PRPr group. For all tests, $\mathrm{P}$ values $<0.05$ were considered statistically significant.

Correlation analysis was performed by means of a Spearman's rho test. All data collected were categorized per animal. In this way we were able to make correlations between pain measurements at different time points and the corresponding histological findings at day 21. For the interpretation of the correlation coefficient we used the absolute value of $\mathrm{r}_{\mathrm{s}}$, dividing the correlations in weak $(<0.5)$, moderate $(0.40-0.59)$, strong $(0.60-0.79)$ and very strong $(>0.80)$. Correlations were significant if $\mathrm{P}$ values $<0.05$. 


\section{Results}

\section{Multiple PRPr injections reduce pain}

We determined the weight distribution over the hind limbs as an indicator of pain (Figure 2A). Seven days after induction of OA, $43.1 \pm 9.6 \%$ of weight was distributed on the affected limb, indicating pain (Figure 2B). Mice in the PRPr group significantly increased the weight on the affected joint on day $7(\mathrm{P}=0.041)$ and $14(\mathrm{P}=0.034)$ compared to day 0 , indicating a reduction in pain. The mice that received saline injections did not significantly change the weight distribution on any of the time points, compared to the start of treatment, albeit a trend for improvement over time was visible. The largest difference between PRPr and saline treated animals was seen at day 7, although this did not reach statistical significance.

\section{Multiple PRPr injections have no effect on cartilage damage}

Collagenase injected knees that were treated with saline (control group) had more cartilage damage than the healthy controls at day 21 for maximum OA scores and at both time points for sum scores, confirming development of OA (Figure 3A, B, P<0.05). PRPr injected knees were not significantly different from the healthy group at both time points regarding maximum OA scores. Regarding sum scores, this was only seen at the later time point. This could suggest a protective effect of the PRPr on cartilage, although direct comparison of cartilage damage between saline and PRPr groups at day 21 showed only a trend towards a protective effect of $\operatorname{PRPr}(\mathrm{P}=0.053)$. 

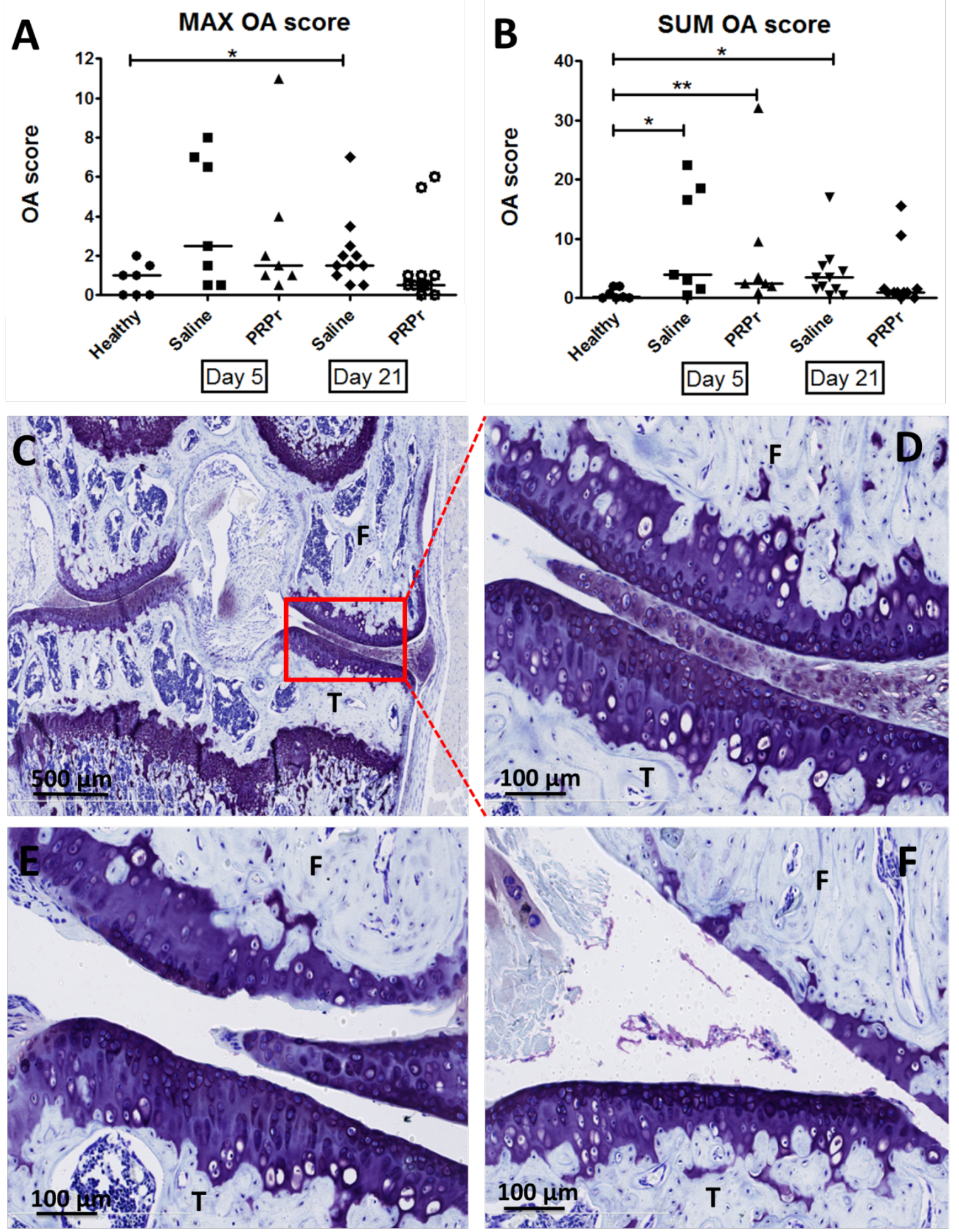

Fig 3 revised, graph B added 


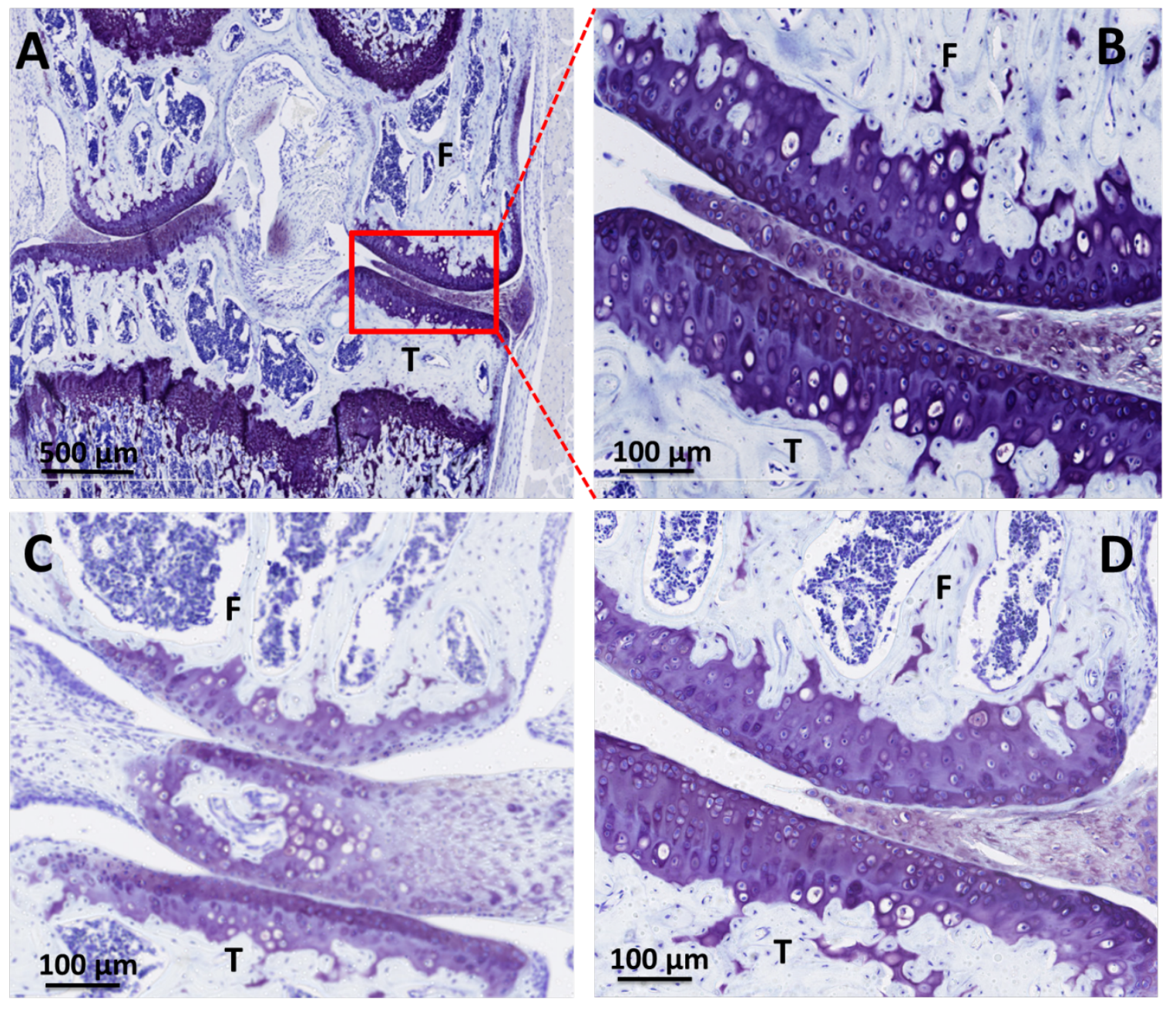

Supp file 1: A-B = healthy (same plaatje als in fig 3, C = Saline, D = PRPr)

\section{Multiple PRPr injections reduce synovial membrane thickening}

Collagenase injected knees displayed a significantly thickened synovial membrane compared to healthy knees. Although the synovial membrane thickness was not statistically different between PRPr and saline injected groups 5 days after start of treatment, synovial membrane thickness at day 21 was significantly less in the PRPr group than in the saline control group (Figure 4A, $\mathrm{P}=0.041$ ). Overall, synovial membrane thickness was largely reduced over time between day 21 and day $5(\mathrm{P}<0.001)$.

\section{Multiple PRPr injections maintain the CD206 and CD163 positive macrophages}

To further analyze the synovial inflammation process, we assessed the presence of different macrophage subtypes. We examined the presence of iNOS, CD206 and CD163 positive 
macrophages by ranking healthy, collagenase injected saline control and collagenase injected PRPr treated knees at day 5 and day 21 between weak, moderate and strong staining intensity (Figure 5A1-C4). The presence of iNOS positive macrophages, indicating a pro-inflammatory response, was higher in collagenase injected knees than in healthy control knees at both day 5 (saline $\mathrm{P}=0.004$; $\mathrm{PRPr} \mathrm{P}=0.006$ ) and day 21 (saline $\mathrm{P}=0.016$; $\mathrm{PRPr} \mathrm{P}=0.046$ ), independent of treatment (Figure 5A). Although no significant differences were observed in the presence of iNOS positive macrophages between treatment groups, PRPr injected knees did show a trend towards less iNOS positive macrophages than in the saline injected knees at day 5 ( $\mathrm{P}=0.109)$. We furthermore determined the presence of macrophages related to tissue repair (CD206+) and anti-inflammatory macrophages (CD163+). In the collagenase injected saline control group, the presence of CD163 and CD206 positive macrophages was significantly lower than in the healthy knees, at day 5 (Figure 5B-C, $\mathrm{P}=0.024$ and $\mathrm{P}=0.042$ respectively). The presence of CD206 and CD163 positive macrophages in the PRPr treated knees did not differ from that of saline treated knees, at day 5 or day 21). In the saline group at day 21, the presence of CD206, but not CD163, was significantly increased compared to day 5 ( $\mathrm{P}=0.023$ and $\mathrm{P}=0.185$ respectively). 

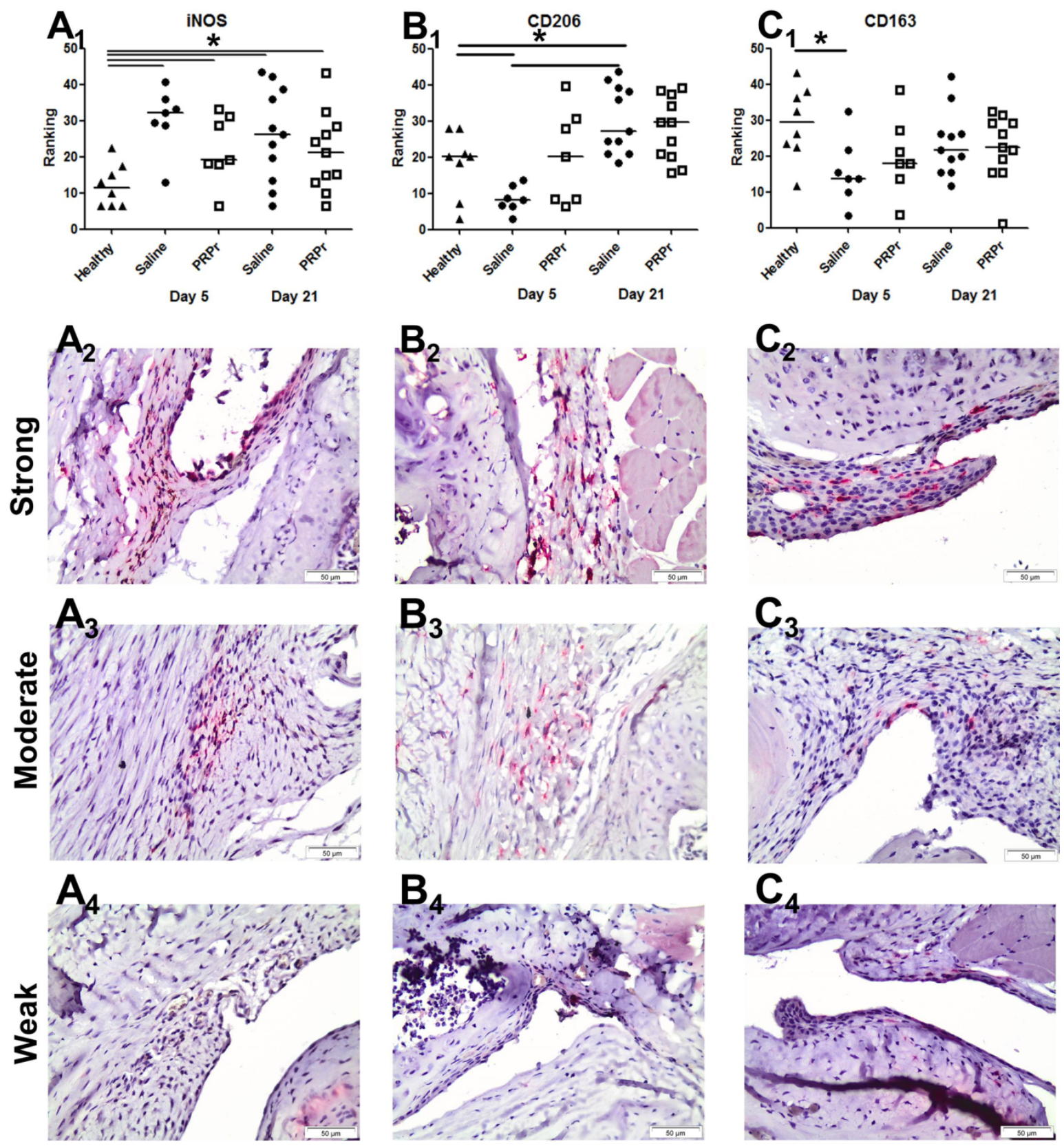

Fig 5 revised, all histology added

\section{Pain reduction is associated with a thinner synovial membrane}

Interestingly, reduction of pain measured at day 7 was strongly associated with a thinner synovial membrane at day 21 within the same animals (Table 1, P=0.002). There was no significant correlation between pain reduction at day 21 and synovial thickness at day 21, possibly due to the overall pain reduction seen in all animals. Furthermore, the presence of iNOS+ macrophages was moderately associated with lateral OA damage $(\mathrm{P}=0.02)$. No significant associations were found 
between iNOS+ macrophages and pain or synovial thickness between treatment groups. The presence of repair macrophages (CD206+) was associated with a thinner synovial membrane $(\mathrm{P}=0.007)$ and anti-inflammatory macrophages $(\mathrm{CD} 163+, \mathrm{P}<0.001)$. 


\section{Discussion}

The results of this study suggest that multiple intra-articular injections of PRPr in a collagenase induced OA (CIOA) mouse model reduce synovial inflammation and might have a protective effect on cartilage, while at the same time reducing pain. The strongest effect on pain reduction was seen in the period shortly after start of treatment. Next to pain reduction, multiple PRPr injections inhibited synovial inflammation, as demonstrated by a thinner synovial membrane compared to the saline control. Furthermore, PRPr injections had an effect on the balance between inflammatory and anti-inflammatory macrophages in the synovial membrane, in particular by preventing the early decrease in anti-inflammatory macrophages seen after induction of CIOA. We also noted that, although the association was not significant, PRPrinjected knees tended to have fewer pro-inflammatory iNOS+ macrophages than saline-injected knees.

Collagenase injections induce joint inflammation, in particular in the first two weeks, making this model suitable for testing potential anti-inflammatory therapies. For example, others have shown that intra-articular injection of adipose-derived stem cells (ASC) in this model reduces synovial inflammation at day 42, when ASCs are injected one week after induction of CIOA. $^{49}$ This demonstrates the possibility to interfere with inflammation using biological treatments in this model. Our results confirm that early intervention in this model can have beneficial effects.

PRPr injections reduced pain for two consecutive weeks. Mice with high pain levels at day 7 were very likely to have thicker synovial membranes two weeks later. The pathway by which PRPr reduces pain may involve inhibition of the production of prostaglandin E2 (PGE2). PGE2 is a lipid mediator of inflammatory pain that causes pain hypersensitivity via nociceptor sensitization. ${ }^{51}$ In an inflammatory environment, the main contributors to PGE2 release are thought to be tissue-resident macrophages. ${ }^{51}$ Our data suggest that PRPr-injected knees may have fewer pro-inflammatory and more anti-inflammatory macrophages, possible resulting in lower PGE2 production. It is known that PRP can promote the differentiation of monocytes towards repair and anti-inflammatory CD206 and CD163-positive macrophages. ${ }^{40}$ This is supported by the finding in a rabbit knee osteoarthritis model that intra-articular injections of leucocyte-poor PRP reduced PGE2 concentrations. ${ }^{57}$ Moreover, PRPr has been reported to contain high levels of interleukin 1 receptor antagonist (IL-1Ra) ${ }^{35}$ that can inhibit acute inflammation caused by IL1 
and promote macrophage polarization towards an M2 phenotype. ${ }^{28}$ Our finding that animals in neither the treated nor the untreated group appeared to experience any pain at three weeks may be partly due to the fact that acute inflammation weakens in time after collagenase injection, thereby reducing nociceptive input to the central nervous system. Less nociceptive input can be preceded by desensitization of the mice nervous system for pain. In the latter case, the threshold for the activation of the joint nociceptors is reduced, and thus a bigger stimulus is needed to register pain. $^{31}$

Besides synovial inflammation, cartilage damage is an important hallmark of OA. In this study, 28 days after OA induction, cartilage damage in the lateral joint compartment after multiple PRPr injections was not different from the healthy knees. The severity of the cartilage damage, however, was significantly increased in the lateral compartment of the CIOA joints treated with saline. The mild cartilage damage we observed in our study made it difficult to detect differences between PRPr and saline groups. The absence of a difference between PRPr treated CIOA mice and healthy mice could be an indication of a chondroprotective effect of PRPr injections. No correlation between pain and cartilage damage at any time point was found, confirming previous findings in the field. ${ }^{32}$

We used a commercially available human derived PRP, which was pooled from 5 healthy human donors. Pooling PRP donors can reduce the inter donor variability described previously. ${ }^{30 \text {, }}$ 56 This PRP is poor in leucocytes and in this study was activated prior to injection in mice. In contrast, others inject non-activated PRP and rely on activation in vivo., 8, 12, ${ }^{41}$ We choose to activate the PRP product prior to injection because firstly, it is hard to control the activation of PRP in vivo, and thus hard to draw conclusions about the working mechanism of PRP without knowing the level of activation. ${ }^{9}$ Some of the disappointing results from other studies might be attributed to less than optimal activation. Secondly, activating PRP with $\mathrm{CaCl}_{2}$ leads to higher levels of PDGF-AA and BB than other activation methods such as freeze-thaw. ${ }^{46}$ Thirdly, the activation of PRP results in the formation of a so-called cloth, which catches any remaining erythrocytes and leucocytes, making the end product low in cells and high in growth factors. Although possible positive effects of a PRP product rich in leucocytes is still being debated ${ }^{2}$, a product depleted from allogenic or in this case xenogeneic cells will cause less immunoreaction. Lastly, by having a PRP product low in leucocytes and short storage before activation, we can reduce the catabolic factors in PRP or PRPr. ${ }^{50}$ Since leucocytes are the main contributors to 
TNF $\alpha$ levels in PRP - but also to levels of interleukins 6 and 8 - these levels might increase further in the period during which leucocyte-rich PRP is stored. ${ }^{10,}{ }^{23}$ Although the working mechanism of PRP is not fully understood, the current knowledge about its active components is improving. This will likely help the PRPr product to be optimized by filtering out components, such as TNF $\alpha$ and vascular endothelial growth factor (VEGF), or increasing the concentrations of other components such as PDGF. Others have reported that freeze drying of PRP into a powder increases its efficacy. Freeze drying could also prolong the lifetime of the PRP product, making it an off-the-shelf product with a longer lifetime. ${ }^{21,}{ }^{36}$ We could therefore reduce the levels of catabolic factors in PRPr by selecting a PRP product low in leucocytes and minimizing the duration of storage before activation. ${ }^{50}$ Nevertheless, clinical studies most regularly use nonactivated PRP, making our results not fully comparable or immediately clinically translatable.

Here we have demonstrated in an OA model that multiple PRPr injections reduce pain and synovial membrane thickness, and that PRPr appears to modulate the phenotype of synovial macrophages. We believe that PRPr injections are a more potent therapy for early stage intervention after trauma and early OA, rather than a treatment for end-stage OA. The latter is confirmed in a few clinical trials, where PRP injections did not affect patients with end-stage OA. ${ }^{18}$ This knowledge can be used in future experiments to determine the best time point for intra-articular PRP injections after trauma, and to further evaluate and confirm the chondroprotective effects of PRP in the long term. Together with improvements of the PRP or PRPr product itself, this could help to make PRP a suitable treatment shorty after joint trauma or for patients with low grade OA, both for pain relief and the inhibition of OA pathophysiology. 


\section{References}

1. Akeda K, Ohishi K, Masuda K, et al. Intradiscal Injection of Autologous Platelet-Rich Plasma Releasate to Treat Discogenic Low Back Pain: A Preliminary Clinical Trial. Asian Spine J. 2017;11(3):380-389.

2. Assirelli E, Filardo G, Mariani E, et al. Effect of two different preparations of platelet-rich plasma on synoviocytes. Knee Surg Sports Traumatol Arthrosc. 2015;23(9):2690-2703.

3. Borbely E, Sandor K, Markovics A, et al. Role of capsaicin-sensitive nerves and tachykinins in mast cell tryptase-induced inflammation of murine knees. Inflamm Res. 2016;65(9):725-736.

4. Brossi PM, Moreira JJ, Machado TS, Baccarin RY. Platelet-rich plasma in orthopedic therapy: a comparative systematic review of clinical and experimental data in equine and human musculoskeletal lesions. BMC Vet Res. 2015;11:98.

5. Bulstra SK, Drukker J, Kuijer R, Buurman WA, van der Linden AJ. Thionin staining of paraffin and plastic embedded sections of cartilage. Biotech Histochem. 1993;68(1):20-28.

6. Campbell KA, Saltzman BM, Mascarenhas R, et al. Does Intra-articular Platelet-Rich Plasma Injection Provide Clinically Superior Outcomes Compared with Other Therapies in the Treatment of Knee Osteoarthritis? A Systematic Review of Overlapping Meta-analyses. Arthroscopy J Arthroscopic Relat Surg. 2015;31(11):2213-2221.

7. Camplejohn KL, Allard SA. Limitations of safranin 'O' staining in proteoglycan-depleted cartilage demonstrated with monoclonal antibodies. Histochemistry. 1988;89(2):185-188.

8. Castelijns G, Crawford A, Schaffer J, Ortolano GA, Beauregard T, Smith RK. Evaluation of a filterprepared platelet concentrate for the treatment of suspensory branch injuries in horses. Vet Comp Orthop Traumatol. 2011;24(5):363-369.

9. Cavallo C, Roffi A, Grigolo B, et al. Platelet-Rich Plasma: The Choice of Activation Method Affects the Release of Bioactive Molecules. Biomed Res Int. 2016;2016:6591717.

10. Chaudhary R, Aggarwal A, Khetan D, Dayal R. Cytokine generation in stored platelet concentrate: comparison of two methods of preparation. Indian J Med Res. 2006;124(4):427-430.

11. Cook JL, Smith PA, Bozynski CC, et al. Multiple injections of leukoreduced platelet rich plasma reduce pain and functional impairment in a canine model of $A C L$ and meniscal deficiency. J Orthop Res. 2016;34(4):607-615.

12. de Vos RJ, Weir A, van Schie HT, et al. Platelet-rich plasma injection for chronic Achilles tendinopathy: a randomized controlled trial. JAMA. 2010;303(2):144-149.

13. Dohan Ehrenfest DM, Rasmusson L, Albrektsson T. Classification of platelet concentrates: from pure platelet-rich plasma (P-PRP) to leucocyte- and platelet-rich fibrin (L-PRF). Trends Biotechnol. 2009;27(3):158-167.

14. Dong J, Jiang D, Wang Z, Wu G, Miao L, Huang L. Intra-articular delivery of liposomal celecoxibhyaluronate combination for the treatment of osteoarthritis in rabbit model. Int J Pharm. 2013;441(1-2):285-290.

15. Fernihough J, Gentry C, Malcangio $M$, et al. Pain related behaviour in two models of osteoarthritis in the rat knee. Pain. 2004;112(1-2):83-93.

16. Glasson SS, Chambers MG, Van Den Berg WB, Little CB. The OARSI histopathology initiative recommendations for histological assessments of osteoarthritis in the mouse. Osteoarthritis Cartilage. 2010;18 Suppl 3:S17-23.

17. Gobbi A, Lad D, Karnatzikos G. The effects of repeated intra-articular PRP injections on clinical outcomes of early osteoarthritis of the knee. Knee Surg Sports Traumatol Arthrosc. 2015;23(8):2170-2177. 
18. Gormeli G, Gormeli CA, Ataoglu B, Colak C, Aslanturk O, Ertem K. Multiple PRP injections are more effective than single injections and hyaluronic acid in knees with early osteoarthritis: a randomized, double-blind, placebo-controlled trial. Knee Surg Sports Traumatol Arthrosc. 2015.

19. Inglis JJ, McNamee KE, Chia SL, et al. Regulation of pain sensitivity in experimental osteoarthritis by the endogenous peripheral opioid system. Arthritis Rheum. 2008;58(10):3110-3119.

20. Kaps C, Loch A, Haisch A, et al. Human platelet supernatant promotes proliferation but not differentiation of articular chondrocytes. Med Biol Eng Comput. 2002;40(4):485-490.

21. Kieb M, Sander F, Prinz C, et al. Platelet-Rich Plasma Powder: A New Preparation Method for the Standardization of Growth Factor Concentrations. Am J Sports Med. 2016.

22. Knop E, Paula LE, Fuller R. Platelet-rich plasma for osteoarthritis treatment. Rev Bras Reumatol Engl Ed. 2016;56(2):152-164.

23. Kobayashi $\mathrm{Y}$, Saita $\mathrm{Y}$, Nishio $\mathrm{H}$, et al. Leukocyte concentration and composition in platelet-rich plasma (PRP) influences the growth factor and protease concentrations. J Orthop Sci. 2016;21(5):683-689.

24. Lacci KM, Dardik A. Platelet-rich plasma: support for its use in wound healing. Yale J Biol Med. 2010;83(1):1-9.

25. Lai LP, Stitik TP, Foye PM, Georgy JS, Patibanda V, Chen B. Use of Platelet-Rich Plasma in IntraArticular Knee Injections for Osteoarthritis: A Systematic Review. PM R. 2015;7(6):637-648.

26. Laudy $A B$, Bakker EW, Rekers $M$, Moen $M H$. Efficacy of platelet-rich plasma injections in osteoarthritis of the knee: a systematic review and meta-analysis. $\mathrm{Br} J$ Sports Med. 2015;49(10):657-672.

27. Lorenz J, Grassel S. Experimental osteoarthritis models in mice. Methods Mol Biol. 2014;1194:401-419.

28. Luz-Crawford P, Djouad F, Toupet $K$, et al. Mesenchymal Stem Cell-Derived Interleukin 1 Receptor Antagonist Promotes Macrophage Polarization and Inhibits B Cell Differentiation. Stem Cells. 2016;34(2):483-492.

29. Marx RE. Platelet-rich plasma: evidence to support its use. J Oral Maxillofac Surg. 2004;62(4):489-496.

30. Mazzocca AD, McCarthy MB, Chowaniec DM, et al. Platelet-rich plasma differs according to preparation method and human variability. J Bone Joint Surg Am. 2012;94(4):308-316.

31. McDougall JJ. Arthritis and pain. Neurogenic origin of joint pain. Arthritis Res Ther. 2006;8(6):220.

32. McDougall JJ, Linton P. Neurophysiology of arthritis pain. Curr Pain Headache Rep. 2012;16(6):485-491.

33. Meheux CJ, McCulloch PC, Lintner DM, Varner KE, Harris JD. Efficacy of Intra-articular PlateletRich Plasma Injections in Knee Osteoarthritis: A Systematic Review. Arthroscopy J Arthroscopic Relat Surg. 2016;32(3):495-505.

34. Montañez-Heredia E, Irízar S, Huertas PJ, et al. Intra-articular injections of platelet-rich plasma versus hyaluronic acid in the treatment of osteoarthritic knee pain: A randomized clinical trial in the context of the Spanish national health care system. Int J Mol Sci. 2016;17(7).

35. Mussano F, Genova T, Munaron L, Petrillo S, Erovigni F, Carossa S. Cytokine, chemokine, and growth factor profile of platelet-rich plasma. Platelets. 2016;27(5):467-471.

36. Nakatani Y, Agata H, Sumita Y, Koga T, Asahina I. Efficacy of freeze-dried platelet-rich plasma in bone engineering. Arch Oral Biol. 2017;73:172-178.

37. Nikolidakis D, Jansen JA. The biology of platelet-rich plasma and its application in oral surgery: literature review. Tissue Eng Part B Rev. 2008;14(3):249-258. 
38. Obata S, Akeda K, Imanishi T, et al. Effect of autologous platelet-rich plasma-releasate on intervertebral disc degeneration in the rabbit anular puncture model: a preclinical study. Arthritis Res Ther. 2012;14(6):R241.

39. Osterman C, McCarthy MB, Cote MP, et al. Platelet-Rich Plasma Increases Anti-inflammatory Markers in a Human Coculture Model for Osteoarthritis. Am J Sports Med. 2015;43(6):14741484.

40. Papait A, Cancedda R, Mastrogiacomo M, Poggi A. Allogeneic platelet-rich plasma affects monocyte differentiation to dendritic cells causing an anti-inflammatory microenvironment putatively fostering the wound healing. J Tissue Eng Regen Med. 2016.

41. Radice F, Yanez R, Gutierrez V, Rosales J, Pinedo M, Coda S. Comparison of magnetic resonance imaging findings in anterior cruciate ligament grafts with and without autologous plateletderived growth factors. Arthroscopy. 2010;26(1):50-57.

42. Sakata R, McNary SM, Miyatake K, et al. Stimulation of the superficial zone protein and lubrication in the articular cartilage by human platelet-rich plasma. Am J Sports Med. 2015;43(6):1467-1473.

43. Sakata R, Reddi AH. Platelet-Rich Plasma Modulates Actions on Articular Cartilage Lubrication and Regeneration. Tissue Eng Part B Rev. 2016;22(5):408-419.

44. Schelbergen RF, van Dalen S, ter Huurne M, et al. Treatment efficacy of adipose-derived stem cells in experimental osteoarthritis is driven by high synovial activation and reflected by S100A8/ A9 serum levels. Osteoarthritis Cartilage. 2014;22(8):1158-1166.

45. Smith PA. Intra-articular Autologous Conditioned Plasma Injections Provide Safe and Efficacious Treatment for Knee Osteoarthritis: An FDA-Sanctioned, Randomized, Double-blind, Placebocontrolled Clinical Trial. Am J Sports Med. 2016;44(4):884-891.

46. Sonker A, Dubey A. Determining the Effect of Preparation and Storage: An Effort to Streamline Platelet Components as a Source of Growth Factors for Clinical Application. Transfus Med Hemother. 2015;42(3):174-180.

47. Spreafico A, Chellini F, Frediani B, et al. Biochemical investigation of the effects of human platelet releasates on human articular chondrocytes. J Cell Biochem. 2009;108(5):1153-1165.

48. Sundman EA, Cole BJ, Karas V, et al. The anti-inflammatory and matrix restorative mechanisms of platelet-rich plasma in osteoarthritis. Am J Sports Med. 2014;42(1):35-41.

49. Ter Huurne M, Schelbergen R, Blattes R, et al. Antiinflammatory and chondroprotective effects of intraarticular injection of adipose-derived stem cells in experimental osteoarthritis. Arthritis Rheum. 2012;64(11):3604-3613.

50. Textor JA, Tablin F. Intra-articular use of a platelet-rich product in normal horses: clinical signs and cytologic responses. Vet Surg. 2013;42(5):499-510.

51. Ulmann L, Hirbec H, Rassendren F. P2X4 receptors mediate PGE2 release by tissue-resident macrophages and initiate inflammatory pain. EMBO J. 2010;29(14):2290-2300.

52. van Buul GM, Koevoet WL, Kops N, et al. Platelet-rich plasma releasate inhibits inflammatory processes in osteoarthritic chondrocytes. Am J Sports Med. 2011;39(11):2362-2370.

53. Van Buul GM, Siebelt M, Leijs MJC, et al. Mesenchymal stem cells reduce pain but not degenerative changes in a mono-iodoacetate rat model of osteoarthritis. J Orthop Res. 2014;32(9):1167-1174.

54. van der Kraan PM, Vitters EL, van Beuningen HM, van de Putte LB, van den Berg WB. Degenerative knee joint lesions in mice after a single intra-articular collagenase injection. A new model of osteoarthritis. J Exp Pathol (Oxford). 1990;71(1):19-31.

55. van Osch GJ, Blankevoort L, van der Kraan PM, et al. Laxity characteristics of normal and pathological murine knee joints in vitro. J Orthop Res. 1995;13(5):783-791. 
56. Weibrich G, Kleis WK, Hafner G, Hitzler WE. Growth factor levels in platelet-rich plasma and correlations with donor age, sex, and platelet count. J Craniomaxillofac Surg. 2002;30(2):97-102.

57. Yin WJ, Xu HT, Sheng JG, et al. Advantages of pure platelet-rich plasma compared with leukocyteand platelet-rich plasma in treating rabbit knee osteoarthritis. Med Sci Monit. 2016;22:12801290.

58. Zhou Q, Xu C, Cheng X, et al. Platelets promote cartilage repair and chondrocyte proliferation via ADP in a rodent model of osteoarthritis. Platelets. 2016;27(3):212-222. 